\title{
A diagnostic model for minimal change disease based on biological parameters
}

\author{
Hanyu Zhu ${ }^{1}$, Qiuxia Han ${ }^{2}$, Dong Zhang ${ }^{\text {Corresp.. }}{ }^{1}$ ， Yong Wang ${ }^{\text {Corresp., }}{ }^{1}$ ， Jing Gao ${ }^{3}$ ， Wenjia Geng ${ }^{1}$, Xiaoli Yang ${ }^{1}$ \\ , Xiangmei Chen ${ }^{1}$ \\ ${ }^{1}$ Department of Nephrology, Chinese PLA General Hospital, Chinese PLA Institute of Nephrology, State Key Laboratory of Kidney Diseases, National \\ Clinical Research Center of Kidney Diseases, Beijing Key Laboratory of Kidney Disease, Beijing, China \\ 2 Department of Nephrology, The First Affiliated Hospital of Zhengzhou University, Zhengzhou, China \\ 3 Department of Clinical Biochemistry, Chinese PLA General Hospital, Beijing, China \\ Corresponding Authors: Dong Zhang, Yong Wang \\ Email address: dongzhang301301@126.com,wangyong301kidney@163.com
}

Background: Minimal change disease (MCD) is a kind of nephrotic syndrome (NS). In this study, we aimed to establish a mathematical diagnostic model based on biological parameters to classify MCD. Methods: A total of 798 NS patients were divided into MCD group and control group. The comparison of biological indicators between two groups were performed with t-tests. Logistic regression was used to establish the diagnostic model, and the diagnostic value of the model was estimated using receiver operating characteristic (ROC) analysis. Results: 13 indicators including Anti-phospholipase $A 2$ receptor (antiPLA2R) $(P=0.000)$, Total protein (TP) $(P=0.000)$, Albumin (ALB) $(P=0.000)$, Direct bilirubin (DB) $(P=0.002)$, Creatinine $(\mathrm{Cr})(P=0.000)$, Total cholesterol $(\mathrm{CH})(P=0.000)$, Lactate dehydrogenase $(\mathrm{LDH})(P=0.007)$, High density lipoprotein cholesterol (HDL) $(P=0.000)$, Low density lipoprotein cholesterol (LDL) $(P=0.000)$, Thrombin time $(T T)(P=0.000)$, Plasma fibrinogen (FIB) $(P=0.000)$, Immunoglobulin A ( $\operatorname{IgA})(P=0.008)$ and Complement 3 (C3) $(P=0.019)$ were significantly correlated with MCD. Furthermore, the area under ROC curves of $\mathrm{CH}, \mathrm{HDL}, \mathrm{LDL}, \mathrm{TT}$ and FIB were more than 0.70. Logistic analysis demonstrated that $\mathrm{CH}$ and $\Pi T$ were risk factors for MCD. According to the ROC of " $\mathrm{CH}+\Pi$ ", the AUC was 0.827, with the sensitivity of $83.0 \%$ and the specificity of $69.8 \%(P=0.000)$. Conclusion: The established diagnostic model with $\mathrm{CH}$ and $\Pi \mathrm{T}$ could be used for classified diagnosis of MCD. 
1 A diagnostic model for minimal change disease based on biological parameters

2

3 Hanyu Zhu 1*, Qiuxia Han 2*, Dong Zhang 1\#, Yong Wang 1\#, Jing Gao 3, Wenjia Geng 1,

4 Xiaoli Yang 1, Xiangmei Chen 1

5

61 Department of Nephrology, Chinese PLA General Hospital, Chinese PLA Institute of

7 Nephrology, State Key Laboratory of Kidney Diseases, National Clinical Research Center of

8 Kidney Diseases, Beijing Key Laboratory of Kidney Disease, Beijing 100853, China

92 Department of Nephrology, The First Affiliated Hospital of Zhengzhou University, 1 East

10 Jianshe Road, Zhengzhou 450052, China

11 12

3 Department of Clinical Biochemistry, Chinese PLA General Hospital, Beijing 100853, China

*Dr. Zhu and Dr. Han contributed equally to this work.

\section{\#Corresponding Author:}

Dong Zhang, Department of Nephrology, Chinese PLA General Hospital, Chinese PLA Institute of Nephrology, State Key Laboratory of Kidney Diseases, National Clinical Research Center for Kidney Diseases, Beijing Key Laboratory of Kidney Disease,28 Fuxing Road, Haidian District, Beijing, 100853, China. Email: dongzhang301301@126.com.

Yong Wang, Department of Nephrology, Chinese PLA General Hospital, Chinese PLA Institute of Nephrology, State Key Laboratory of Kidney Diseases, National Clinical Research Center for Kidney Diseases, Beijing Key Laboratory of Kidney Disease, 28 Fuxing Road, Haidian District, Beijing, 100853, China. Email:wangyong301kidney@163.com 


\section{Abstract}

27 Background: Minimal change disease (MCD) is a kind of nephrotic syndrome (NS). In this 28 study, we aimed to establish a mathematical diagnostic model based on biological parameters to 29 classify MCD.

Methods: A total of 798 NS patients were divided into MCD group and control group. The comparison of biological indicators between two groups were performed with t-tests. Logistic regression was used to establish the diagnostic model, and the diagnostic value of the model was estimated using receiver operating characteristic (ROC) analysis.

Results: 13 indicators including Anti-phospholipase A2 receptor (anti-PLA2R) $(P=0.000)$, Total protein (TP) $(P=0.000)$, Albumin (ALB) $(P=0.000)$, Direct bilirubin (DB) $(P=0.002)$, Creatinine $(\mathrm{Cr})(P=0.000)$, Total cholesterol $(\mathrm{CH})(P=0.000)$, Lactate dehydrogenase $(\mathrm{LDH})(P=0.007)$, High density lipoprotein cholesterol (HDL) $(P=0.000)$, Low density lipoprotein cholesterol (LDL) $(P=0.000)$, Thrombin time (TT) $(P=0.000)$, Plasma fibrinogen (FIB) $(P=0.000)$, Immunoglobulin A (IgA) $(P=0.008)$ and Complement 3 (C3) $(P=0.019)$ were significantly correlated with MCD. Furthermore, the area under ROC curves of CH, HDL, LDL, TT and FIB were more than 0.70. Logistic analysis demonstrated that $\mathrm{CH}$ and $\mathrm{TT}$ were risk factors for MCD. According to the ROC of "CH+TT", the AUC was 0.827 , with the sensitivity of $83.0 \%$ and the specificity of $43 \quad 69.8 \%(P=0.000)$.

44 Conclusion: The established diagnostic model with $\mathrm{CH}$ and $\mathrm{TT}$ could be used for classified diagnosis of MCD. 


\section{Introduction}

Minimal change disease (MCD) is a common pathological type of nephrotic syndrome (NS), and its typical characteristic is the diffuse effacement of podocyte foot processes observed by electron microscope (Glick 2007) The actin of foot process is linked with slit diaphragm, which is important for renal glomerular filtration function. MCD is commonly seen in child patients with NS. According to the reports, about $80 \%$ MCD cases are aged less than 10 years old. The incidence of MCD in adults is lower than that in children (Cameron 1996; Haas et al. 1997; Kazi et al. 2009; Zech et al. 1982). In our country, the incidence of adult MCD patients in NS patients is less than 25\% (Chu et al. 2014; Zhou et al. 2011). At present, renal biopsy, which is an invasive examination, is required for most glomerulopathy dia gnoses. Although it can offer the diagnosis and testing information for the doctors, renal biopsy may cause complications, such as bleeding. Moreover, some patients can not accept the renal biopsy, leading to the absence of timely diagnosis (Fiorentino et al. 2016; Magistroni et al. 2015; Verde et al. 2012). Therefore, a non-invasive model is urgently needed to discriminate MCD.

It is reported that mathematical model, like classification and regression tree (CART) model can be used as a method to classify different diseases (Hu et al. 2011b; Yan et al. 2011). At present time, the diagnostic model based on data analysis has become the focus of disease diagnosis, and it also can be used in the noninvasive diagnosis (Azmak et al. 2015). Moreover, some reports have shown that the classification equations have been used in kidney diseases.

In present study, with the purpose of classifying MCD and other kidney diseases, we established a diagnostic model based on the clinical parameter. Additionally, we also conducted common statistical analyses, including Chi-square tests, logistic analysis and receiver operating characteristic (ROC) analysis.

\section{Methods}

Study object

This study was approved by the Medical Ethics Committee of the Chinese PLA General Hospital, and the written consents were obtained from all patients. The inclusion criteria of the current research were listed as follows: (1) all the participants were the first time to be admitted into the Department of Nephrology of our hospital; (2) adult patients; (3) no one accepted the renal biopsy before entering our hospital; (4) no one accepted any treatments, including hypertension treatment or hyperlipidemia treatment; (5) no one suffered from any tumors, except hypertension, diabetes, hepatitis or lupus erythematosus; (6) all patients accepted renal biopsy during their hospitalization. The following exclusion criteria were applied in our study: (1) the patients could 
83

not accept renal biopsy; (2) no complete clinical data were provided. According to the inclusion and exclusion criteria, 798 patients were finally recruited, containing 47 MCD patients and 751 patients with other kidney diseases.

\section{Samples and biological parameters}

For all the 798 patients, their blood samples were collected on the second day after entering hospital. Then, blood coagulation test, blood routine examination and clinical biochemistry testing were performed. The demographic data as well as clinical and laboratory examination of all patients were recorded, including age, gender, presence of other diseases, physical examination, and so on.

\section{Statistical analysis}

In this study, all statistical analyses were performed using SPSS 19.0 and GraphPad Prism 5. The data were summarized and presented as means \pm SD. The biological indicators of the two groups were assessed by using t-tests. Logistic regression was employed to establish the diagnostic model. The diagnostic value of the constructed model was examined via performing ROC analysis. $P$ values less than 0.05 were considered to be statistically significant in this paper.

\section{Results}

The characteristics of patients

The tested biological parameters were all listed in Table 1, including Anti-phospholipase A2 receptor (anti-PLA2R), Alanine aminotransferase (ALT), Aspartate aminotransferase (AST), Total protein (TP), Albumin (ALB), Total bilirubin (TB), Direct bilirubin (DB), Alkaline phosphatase (ALP), $\gamma$-Glutamyltransferase (GGT), Glucose (GLU), Urea nitrogen (UN), Creatinine (Cr), Uric acid (Ua), Total cholesterol (CH), Triglyceride (TG), Creatine kinase (CK), Lactate dehydrogenase (LDH), High density lipoprotein cholesterol (HDL), Low density lipoprotein cholesterol (LDL), Thrombin time (TT), Prothrombin time (PT), Plasma fibrinogen (FIB), D-dimer (D2), Immunoglobulin A (IgA), Immunoglobulin G (IgG), Immunoglobulin M (IgM), Immunoglobulin E (IgE), Complement 3 (C3), Complement 4 (C4) and Body mass index (BMI). Moreover, the reference ranges of them were also listed in the table. The demographic data and history of diseases of these two groups were recorded in Table 2. We found that the rates of hypertension and diabetes were declined in MCD patients compared with the patients with other kidney diseases. In MCD group, the numbers of patients less than 40 years old and more than 40 years old were about the same, and the similar result was found in the group of other kidney diseases. We observed more male patients than female patients in both groups. 
119

120

121

122

123

124

125

126

127

128

129

130

131

132

133

134

135

136

137

138

139

140

141

142

143

144

145

146

147

148

149

150

151

152

153

154

Besides, most patients of the two groups had no hypertension, diabetes or hepatitis. The BMI value was $25.03 \pm 4.66$ in MCD group, and the data for the group of other kidney disease was $25.45 \pm 4.37$.

The comparison of biochemical indicators between two groups

In order to explore the association between biochemical indicators and MCD, student's t-test was performed. As shown in Table 3, the results showed that among the 28 biochemical indicators, 13 indicators including anti-PLA2R $(P=0.000)$, TP $(P=0.000)$, ALB $(P=0.000)$, DB $(P=0.002)$, $\mathrm{Cr}(P=0.000), \mathrm{CH}(P=0.000), \mathrm{LDH}(P=0.007), \mathrm{HDL}(P=0.000)$, LDL $(P=0.000)$, TT $(P=0.000)$, FIB $(P=0.000), \operatorname{IgA}(P=0.008)$ and $\mathrm{C} 3(P=0.019)$ were significantly different between the two groups.

ROC analysis of related characteristics

The ROC analysis was conducted to detect the diagnostic value of these 13 indicators, and the results were displayed in Figure 1. We found that in Figure 1, the area under the curves (AUCs) of CH, HDL, LDL, TT and FIB were more than 0.70, and the AUCs of them were 0.807, 0.746, $0.776,0.817$ and 0.713 , respectively $(P=0.032,0.037,0.039,0.032$, and 0.046 , respectively).

\section{Logistic analysis of the pre-selected parameters}

In order to establish the classification models of MCD and other kidney diseases, the logistic analysis was carried out. From Table 4, we could see that $\mathrm{CH}$ and TT were risk factors for MCD, and the $P$ values of them were both 0.000 . Furthermore, the classification equation including $\mathrm{CH}$ and TT was as follows:

$\mathrm{PRE}=1 / 1+\mathrm{e}^{-(10.617-0.270 \times \mathrm{CH}-0.325 \times \mathrm{TT})}$

Then, based on logistic regression of the predicted probability (PRE), the ROC curve of "CH+TT" is presented in Figure 2. From Figure 2, we could see that the AUC of "CH+TT" was 0.827 , with the sensitivity of $83.0 \%$ and the specificity of $69.8 \%(P=0.000)$.

\section{Discussion}

MCD is a kind of glomerular disease caused by lesions of the podocyte. Most MCDs are idiopathic nephrotic syndromes among children and adults. MCD is characterized by hypoalbuminemia, hyperlipidemia, proteinuria and edema (Braden et al. 2000; McGrogan et al. 2011). It has been reported that the outcome of MCD is correlated with some elements, such as virus infection, drugs, allergy and even tumors (Korzets et al. 1992; Meyrier et al. 1992). At present, the pathogenesis of MCD still remains unclear, but many scholars consider that it may 
155

156

157

158

159

160

161

162

163

164

165

166

167

168

169

170

171

172

173

174

175

176

177

178

179

180

181

182

183

184

185

186

187

188

189

190

be associated with podocyte injury. Now in clinic, renal biopsy centesis is still the golden standard for the diagnosis of nephropathy (Appel \& Appel 2009; Floege \& Eitner 2011). Renal biopsy centesis is safe and easy operation, but it is invasive with risks (Fisi et al. 2012). Because some patients have suffered from absolute and relative contraindications of renal biopsy (Mohamed \& John 2011; Whittier \& Korbet 2004), they refuse to undergo renal biopsy. Since the conditions of hospital are not adequate, renal biopsy can not be implemented in every patient who is suspected to have kidney diseases. Moreover, the histopathological diagnosis of renal biopsy is not stable (Lu et al. 2011). Consequently, the non-invasive methods with high accuracy are urgently needed for MCD diagnosis are needed.

The mathematical model based on the statistical analysis and computer technique has been used in clinic, such as computed tomography (CT) and nuclear magnetic resonance (NMR) (Bandak et al. 1995; Hu et al. 2011a). Levery et al. established a new equation of estimated glomerular filtration rate (eGFR), named CKD-EPI, which could assess the stages of kidney diseases (CKD stage) (Levey et al. 2009). Jin et al. also established a diagnostic model for IgA nephropathy with 6 serum biochemical parameters, including ALB, CK, Cr, HDL, CA125 and TB (Gao et al. 2011). Additionally, they certified that this model could be used in the auxiliary diagnosis of $\operatorname{IgA}$ nephropathy.

In our study, a diagnostic model was established for MCD, and the enrolled patients were divided into two groups, which were MCD group and group of other kidney diseases. The present paper enrolled $47 \mathrm{MCD}$ patients and 751 patients with other kidney diseases. In the two groups, the numbers of patients older than 40 years old and younger than 40 years old were almost equal. The ratio of male patients and female patients was about 2:1 in both groups, and very few patients suffered from hypertension, diabetes or hepatitis. Besides, the BMI of the two groups also had no significant differences. The t-test was implemented to analyze the serum biological indicators, and anti-PLA2R, TP, ALB, DB, Cr, CH, LDH, HDL, LDL, TT, FIB, IgA and C3 were significantly different between the two groups. Like the previous study by Gao and his colleagues, which also found 15 significant different serological indicators between IgAN patients and non-IgAN patients, the ROC analysis was performed to further assess the diagnostic value of the collected parameters (Gao et al. 2012). In our study, ROC analysis for the 13 indicators revealed that the AUCs of 5 indicators, $\mathrm{CH}$, HDL, LDL, TT and FIB were more than 0.70. Logistic analysis was performed with $\mathrm{CH}, \mathrm{HDL}, \mathrm{LDL}, \mathrm{TT}$ and FIB. The results suggested that $\mathrm{CH}$ and $\mathrm{TT}$ were risk factors for MCD. Based on logistic regression of the predicted probability on the 2 indicators, the results of ROC curve revealed that " $\mathrm{CH}+\mathrm{TT}$ " the AUC of "CH+TT" was 0.827 , with the sensitivity of $83.0 \%$ and the specificity of $69.8 \%$.

In this study, we found that the combined diagnostic value of " $\mathrm{CH}+\mathrm{TT}$ " was significantly higher than either of them alone. The combined diagnostic model might help improve the diagnosis of 
191

192

193

194

195

196

197

198

199

200

201

202

203

204

205

206

207

208

209

210

211

212

213

214

215

216

MCD, especially for those challenging case. However, the present study has some limitations. Firstly, the sample size of MCD group is not large enough. Moreover, all the patients were collected from a single institution and population, which might cause bias to the final results. Secondly, the specificity was not high in our study, leading to high false positive rate. The diagnostic accuracy of the combined model was $70.4 \%$. In order to improve the diagnosis specificity, we could prioritize diagnosis sensitivity based on the trade-off between diagnosis false positive cases versus omitting true positive cases. According to ROC analysis, the diagnostic specificity was adjusted as $70 \%$, and the sensitivity was $80.9 \%$, while the diagnostic accuracy was $70 \%$, which also hold great potential for clinical application. Thirdly, the diagnostic performance of the constructed models was only verified in the populations collected in the original analysis. A cross-validation was not set to investigate the diagnostic performance of the combined model for MCD in clinic. Additionally, all the MCD cases collected in our study were adults. However, MCD is frequently diagnosed among children, and the adult cases only account for about 10\%-15\%. The main reasons for childhood MCD include congenital anomalies and inherited disorders, but the diagnostic value of the combined model for the childhood MCD cases remained unknown (Downie et al. 2017; Ingelfinger et al. 2016). The distinct etiologies may lead to various clinical symptoms, biological parameters, and therapeutic responses. Thus, further investigations are needed to determinate whether the selected biological parameters exerted advantages in diagnosis of MCD among children. Further related researches will carried to address the above issues.

\section{Conclusion}

This study have established a diagnostic model based on the clinical parameters to classify MCD and other kidney diseases. The combined diagnostic model with $\mathrm{CH}$ and TT could effectively distinguish MCD from other nephrotic syndrome. 
218

219

220

221

222

223

224

225

226

227

228

229

230

231

232

233

234

235

236

237

238

239

240

241

242

243

244

245

246

247

248

249

250

251

252

253

254

255

256

257

258

\section{References}

Appel AS, and Appel GB. 2009. An update on the use of mycophenolate mofetil in lupus nephritis and other primary glomerular diseases. Nat Clin Pract Nephrol 5:132-142. 10.1038/ncpneph1036

Azmak O, Bayer H, Caplin A, Chun M, Glimcher P, Koonin S, and Patrinos A. 2015. Using Big Data to Understand the Human Condition: The Kavli HUMAN Project. Big Data 3:173188. 10.1089/big.2015.0012

Bandak FA, Vander Vorst MJ, Stuhmiller LM, Mlakar PF, Chilton WE, and Stuhmiller JH. 1995. An imaging-based computational and experimental study of skull fracture: finite element model development. J Neurotrauma 12:679-688. 10.1089/neu.1995.12.679

Braden GL, Mulhern JG, O'Shea MH, Nash SV, Ucci AA, Jr., and Germain MJ. 2000. Changing incidence of glomerular diseases in adults. Am J Kidney Dis 35:878-883.

Cameron JS. 1996. Nephrotic syndrome in the elderly. Semin Nephrol 16:319-329.

Chu F, Chen G, and Liu Y. 2014. Pathological patterns of primary nephrotic syndrome in Central China: a retrospective study of 627 cases. Ren Fail 36:514-519. 10.3109/0886022X.2014.882736

Downie ML, Gallibois C, Parekh RS, and Noone DG. 2017. Nephrotic syndrome in infants and children: pathophysiology and management. Paediatr Int Child Health:1-11. 10.1080/20469047.2017.1374003

Fiorentino M, Bolignano D, Tesar V, Pisano A, Van Biesen W, D'Arrigo G, Tripepi G, and Gesualdo L. 2016. Renal Biopsy in 2015--From Epidemiology to Evidence-Based Indications. Am J Nephrol 43:1-19. 10.1159/000444026

Fisi V, Mazak I, Degrell P, Halmai R, Molnar GA, Feher E, Nemeth K, Pinter I, Kovacs T, and Wittmann I. 2012. Histological diagnosis determines complications of percutaneous renal biopsy: a single-center experience in 353 patients. Kidney Blood Press Res 35:26-34. 10.1159/000329939

Floege J, and Eitner F. 2011. Current therapy for IgA nephropathy. J Am Soc Nephrol 22:17851794. 10.1681/ASN.2011030221

Gao J, Cui J, Wang Y, Dong Z, Tian Y, and Xu Y. 2011. Identification of potential predictors for subtype IgA nephropathy through analyses of blood biochemical indicators. Clin Chim Acta 412:441-445. 10.1016/j.cca.2010.11.020

Gao J, Wang Y, Dong Z, Yan Z, Jia X, and Tian Y. 2012. A novel differential diagnostic model based on multiple biological parameters for immunoglobulin A nephropathy. BMC Med Inform Decis Mak 12:58. 10.1186/1472-6947-12-58

Glick AM. 2007. Focal segmental glomerulosclerosis: a case study with review of pathophysiology. Nephrol Nurs J 34:176-182.

Haas M, Meehan SM, Karrison TG, and Spargo BH. 1997. Changing etiologies of unexplained adult nephrotic syndrome: a comparison of renal biopsy findings from 1976-1979 and 1995-1997. Am J Kidney Dis 30:621-631.

$\mathrm{Hu}$ J, Qiao J, Kang D, and Liu B. 2011a. Analysis on the distinguishing features of traditional Chinese therapeutics and related statistical issues. Front Med 5:203-207. 


\subsection{7/s11684-011-0138-6}

Hu W, O'Leary RA, Mengersen K, and Low Choy S. 2011b. Bayesian classification and regression trees for predicting incidence of cryptosporidiosis. PLoS One 6:e23903. 10.1371/journal.pone.0023903

Ingelfinger JR, Kalantar-Zadeh K, and Schaefer F. 2016. Averting the Legacy of Kidney Disease: Focus on Childhood. Int J Organ Transplant Med 7:61-68.

Kazi JI, Mubarak M, Ahmed E, Akhter F, Naqvi SA, and Rizvi SA. 2009. Spectrum of glomerulonephritides in adults with nephrotic syndrome in Pakistan. Clin Exp Nephrol 13:38-43. 10.1007/s10157-008-0075-0

Korzets Z, Golan E, Manor Y, Schneider M, and Bernheim J. 1992. Spontaneously remitting minimal change nephropathy preceding a relapse of Hodgkin's disease by 19 months. Clin Nephrol 38:125-127.

Levey AS, Stevens LA, Schmid CH, Zhang YL, Castro AF, 3rd, Feldman HI, Kusek JW, Eggers P, Van Lente F, Greene T, and Coresh J. 2009. A new equation to estimate glomerular filtration rate. Ann Intern Med 150:604-612.

Lu J, Tam LS, Lai FM, Kwan BC, Choi PC, Li EK, Chow KM, Li PK, and Szeto CC. 2011. Repeat renal biopsy in lupus nephritis: a change in histological pattern is common. $\mathrm{Am} J$ Nephrol 34:220-225. 10.1159/000330356

Magistroni R, D'Agati VD, Appel GB, and Kiryluk K. 2015. New developments in the genetics, pathogenesis, and therapy of IgA nephropathy. Kidney Int 88:974-989. $10.1038 / \mathrm{ki} .2015 .252$

McGrogan A, Franssen CF, and de Vries CS. 2011. The incidence of primary glomerulonephritis worldwide: a systematic review of the literature. Nephrol Dial Transplant 26:414-430. 10.1093/ndt/gfq665

Meyrier A, Delahousse M, Callard P, and Rainfray M. 1992. Minimal change nephrotic syndrome revealing solid tumors. Nephron 61:220-223.

Mohamed N, and John R. 2011. Use of renal biopsy in the elderly. Int Urol Nephrol 43:593-600. 10.1007/s11255-010-9874-9

Verde E, Quiroga B, Rivera F, and Lopez-Gomez JM. 2012. Renal biopsy in very elderly patients: data from the Spanish Registry of Glomerulonephritis. Am J Nephrol 35:230237. $10.1159 / 000336307$

Whittier WL, and Korbet SM. 2004. Renal biopsy: update. Curr Opin Nephrol Hypertens 13:661-665.

Yan FR, Lin JG, and Liu Y. 2011. Sparse logistic regression for diagnosis of liver fibrosis in rat by using SCAD-penalized likelihood. J Biomed Biotechnol 2011:875309. 10.1155/2011/875309

Zech P, Colon S, Pointet P, Deteix P, Labeeuw M, and Leitienne P. 1982. The nephrotic syndrome in adults aged over 60: etiology, evolution and treatment of 76 cases. Clin Nephrol 17:232-236.

Zhou FD, Shen HY, Chen M, Liu G, Zou WZ, Zhao MH, and Wang HY. 2011. The renal histopathological spectrum of patients with nephrotic syndrome: an analysis of 1523 
patients in a single Chinese centre. Nephrol Dial Transplant 26:3993-3997.

301 $10.1093 / \mathrm{ndt} / \mathrm{gfr} 166$

302

303 


\section{Table 1 (on next page)}

The biological parameters in this study

The tested biological parameters. 
1 Table 1. The biological parameters in this study

\begin{tabular}{|c|c|c|}
\hline Index full name & Abbreviation & Reference range \\
\hline Anti-phospholipase A2 receptor & Anti-PLA2R & \\
\hline Alanine aminotransferase & ALT & $0-40 \mathrm{U} / \mathrm{L}$ \\
\hline Aspartate aminotransferase & AST & $0-40 \mathrm{U} / \mathrm{L}$ \\
\hline Total protein & TP & $55-80 \mathrm{~g} / \mathrm{L}$ \\
\hline Albumin & ALB & $35-50 \mathrm{~g} / \mathrm{L}$ \\
\hline Total bilirubin & TB & $0-21 \mu \mathrm{mol} / \mathrm{L}$ \\
\hline Direct bilirubin & DB & $0-8.6 \mu \mathrm{mol} / \mathrm{L}$ \\
\hline Alkaline phosphatase & ALP & $0-130 \mathrm{U} / \mathrm{L}$ \\
\hline Y-Glutamyltransferase & GGT & $0-50 \mathrm{U} / \mathrm{L}$ \\
\hline Glucose & GLU & $3.4-6.2 \mathrm{mmol} / \mathrm{L}$ \\
\hline Urea nitrogen & UN & $1.8-7.5 \mathrm{mmol} / \mathrm{L}$ \\
\hline Creatinine & $\mathrm{Cr}$ & $30-110 \mu \mathrm{mol} / \mathrm{L}$ \\
\hline Uric acid & Ua & $104-444 \mu \mathrm{mol} / \mathrm{L}$ \\
\hline Total cholesterol & $\mathrm{CH}$ & $3.1-5.7 \mathrm{mmol} / \mathrm{L}$ \\
\hline Triglyceride & TG & $0.4-1.7 \mathrm{mmol} / \mathrm{L}$ \\
\hline Creatine kinase & $\mathrm{CK}$ & $2-200 \mathrm{U} / \mathrm{L}$ \\
\hline Lactate dehydrogenase & LDH & $40-250 \mathrm{U} / \mathrm{L}$ \\
\hline High density lipoprotein cholesterol & $\mathrm{HDL}$ & $1-1.6 \mathrm{mmol} / \mathrm{L}$ \\
\hline Low density lipoprotein cholesterol & LDL & $0-3.4 \mathrm{mmol} / \mathrm{L}$ \\
\hline Thrombin time & TT & $16.0-18.0 \mathrm{~s}$ \\
\hline
\end{tabular}




$\begin{array}{lcc}\text { Prothrombin time } & \text { PT } & 11.0-15.0 \mathrm{~s} \\ \text { Plasma fibrinogen } & \text { FIB } & 200-400 \mathrm{mg} / \mathrm{dL} \\ \text { D-dimer } & \text { D2 } & 0.0-0.5 \mu \mathrm{g} / \mathrm{L} \\ \text { Immunoglobulin A } & \text { IgA } & 70-180 \mathrm{mg} / \mathrm{dl} \\ \text { Immunoglobulin G } & \text { IgG } & 700-1600 \mathrm{mg} / \mathrm{dl} \\ \text { Immunoglobulin M } & \text { IgM } & 40-230 \mathrm{mg} / \mathrm{dl} \\ \text { Immunoglobulin E } & \text { IgE } & 0-100 \mathrm{IU} / \mathrm{ml} \\ \text { Complement 3 } & \text { C3 } & 90-180 \mathrm{mg} / \mathrm{dl} \\ \text { Complement } 4 & \text { C4 } & 10-40 \mathrm{mg} / \mathrm{dl} \\ \text { Body mass index } & \text { BMI } & 18.5-24.99\end{array}$

2

3

4 
Table 2 (on next page)

Basic information of the two groups

The demographic data and history of diseases of these two groups. 
1 Table 2. Basic information of the two groups

\begin{tabular}{cccc}
\hline & MCD group $(\mathrm{n}=47)$ & Group of other kidney diseases $(\mathrm{n}=751)$ & $P$ value \\
\hline Age & & 357 & 0.452 \\
$<40$ & 25 & 394 & \\
$\geq 40$ & 22 & & 0.685 \\
Gender & 30 & 457 & \\
Male & 17 & 294 & 0.000 \\
Female & & & \\
Hypertension & 7 & 363 & 0.041 \\
Yes & 40 & 388 & \\
No & & & \\
Diabetes & 2 & 113 & 0.050 \\
Yes & 45 & 638 & \\
No & & 57 & 0.895 \\
Hepatitis & 47 & 694 & \\
Yes & $25.03 \pm 4.66$ & $25.45 \pm 4.37$ & \\
No & & & \\
BMI & & 578
\end{tabular}

2

3

4 


\section{Table 3 (on next page)}

The comparison of serological parameters in the two groups 
1 Table 3. The comparison of serological parameters in the two groups

\begin{tabular}{|c|c|c|c|}
\hline Parameter & $\begin{array}{l}\text { MCD group }(n=47) \\
\quad(\text { mean } \pm S D)\end{array}$ & $\begin{array}{c}\text { Group of other kidney } \\
\text { diseases }(n=751)(\text { mean } \pm S D)\end{array}$ & $P$ value \\
\hline Anti-PLA2R & $2.00 \pm 0.02$ & $34.22 \pm 114.96$ & $0.000^{*}$ \\
\hline ALT & $27.00 \pm 35.12$ & $22.61 \pm 21.99$ & 0.201 \\
\hline AST & $20.79 \pm 8.80$ & $18.82 \pm 11.39$ & 0.240 \\
\hline $\mathrm{TP}$ & $43.32 \pm 10.01$ & $58.33 \pm 12.02$ & $0.000^{*}$ \\
\hline ALB & $22.68 \pm 7.87$ & $33.94 \pm 9.04$ & $0.000^{*}$ \\
\hline TB & $7.87 \pm 3.64$ & $8.63 \pm 4.40$ & 0.177 \\
\hline DB & $1.39 \pm 0.99$ & $1.98 \pm 1.32$ & $0.002^{*}$ \\
\hline ALP & $69.36 \pm 34.22$ & $66.03 \pm 31.66$ & 0.835 \\
\hline GGT & $41.43 \pm 82.16$ & $34.00 \pm 51.21$ & 0.398 \\
\hline GLU & $4.62 \pm 0.88$ & $4.98 \pm 1.75$ & 0.102 \\
\hline UN & $6.78 \pm 4.30$ & $6.30 \pm 3.59$ & 0.446 \\
\hline $\mathrm{Cr}$ & $82.25 \pm 23.15$ & $104.31 \pm 68.19$ & $0.000^{*}$ \\
\hline Ua & $345.71 \pm 104.69$ & $366.78 \pm 102.00$ & 0.198 \\
\hline $\mathrm{CH}$ & $8.81 \pm 3.23$ & $5.72 \pm 2.21$ & $0.000^{*}$ \\
\hline TG & $2.35 \pm 1.60$ & $2.22 \pm 1.56$ & 0.582 \\
\hline $\mathrm{CK}$ & $97.12 \pm 88.43$ & $95.70 \pm 99.36$ & 0.756 \\
\hline LDH & $204.66 \pm 58.78$ & $182.88 \pm 59.31$ & $0.007^{*}$ \\
\hline $\mathrm{HDL}$ & $1.74 \pm 0.76$ & $1.26 \pm 0.84$ & $0.000^{*}$ \\
\hline LDL & $6.31 \pm 2.81$ & $3.81 \pm 1.84$ & $0.000^{*}$ \\
\hline TT & $18.65 \pm 2.52$ & $16.56 \pm 1.44$ & $0.000^{*}$ \\
\hline PT & $12.85 \pm 0.83$ & $13.15 \pm 1.55$ & 0.243 \\
\hline FIB & $5.46 \pm 1.79$ & $4.22 \pm 1.50$ & $0.000^{*}$ \\
\hline D2 & $1.07 \pm 0.79$ & $0.98 \pm 1.84$ & 0.721 \\
\hline $\lg A$ & $220.47 \pm 92.70$ & $259.46 \pm 117.18$ & $0.008^{*}$ \\
\hline $\lg M$ & $142.22 \pm 64.44$ & $111.43 \pm 126.53$ & 0.099 \\
\hline $\lg E$ & $445.22 \pm 927.74$ & $205.44 \pm 894.09$ & 0.090 \\
\hline C3 & $116.86 \pm 27.12$ & $107.55 \pm 26.38$ & $0.019^{*}$ \\
\hline $\mathrm{C} 4$ & $28.15 \pm 10.12$ & $26.46 \pm 9.49$ & 0.238 \\
\hline
\end{tabular}

$2{ }^{*} P<0.05$. 


\section{Table 4(on next page)}

The multivariate logistic regression analysis for the model 
1 Table 4. The multivariate logistic regression analysis for the model

\begin{tabular}{ccccccccc}
\hline & \multirow{2}{*}{$\mathrm{B}$} & S.E. & Wald & df & Sig. & Exp (B) & \multicolumn{2}{c}{$95 \% \mathrm{Cl}$} \\
\cline { 5 - 9 } & & & & & & & & \\
$\mathrm{CH}$ & -.270 & .058 & 21.720 & 1 & .000 & .763 & .682 & .855 \\
$\mathrm{HDL}$ & -.186 & .099 & 3.516 & 1 & .061 & .830 & .684 & 1.008 \\
TT & -.325 & .087 & 14.145 & 1 & .000 & .722 & .610 & .856 \\
Constant & 10.617 & 1.449 & 53.669 & 1 & .000 & 40819.656 & & \\
\hline
\end{tabular}

2

3

4 
Figure 1

The ROC curves of anti-PLA2R, TP, ALB, DB, Cr, CH, LDH, HDL, LDL, TT, FIB, IgA and C3, the related indicators of $M C D$.

The ROC analysis was conducted to detect the diagnostic value of these 13 indicators.

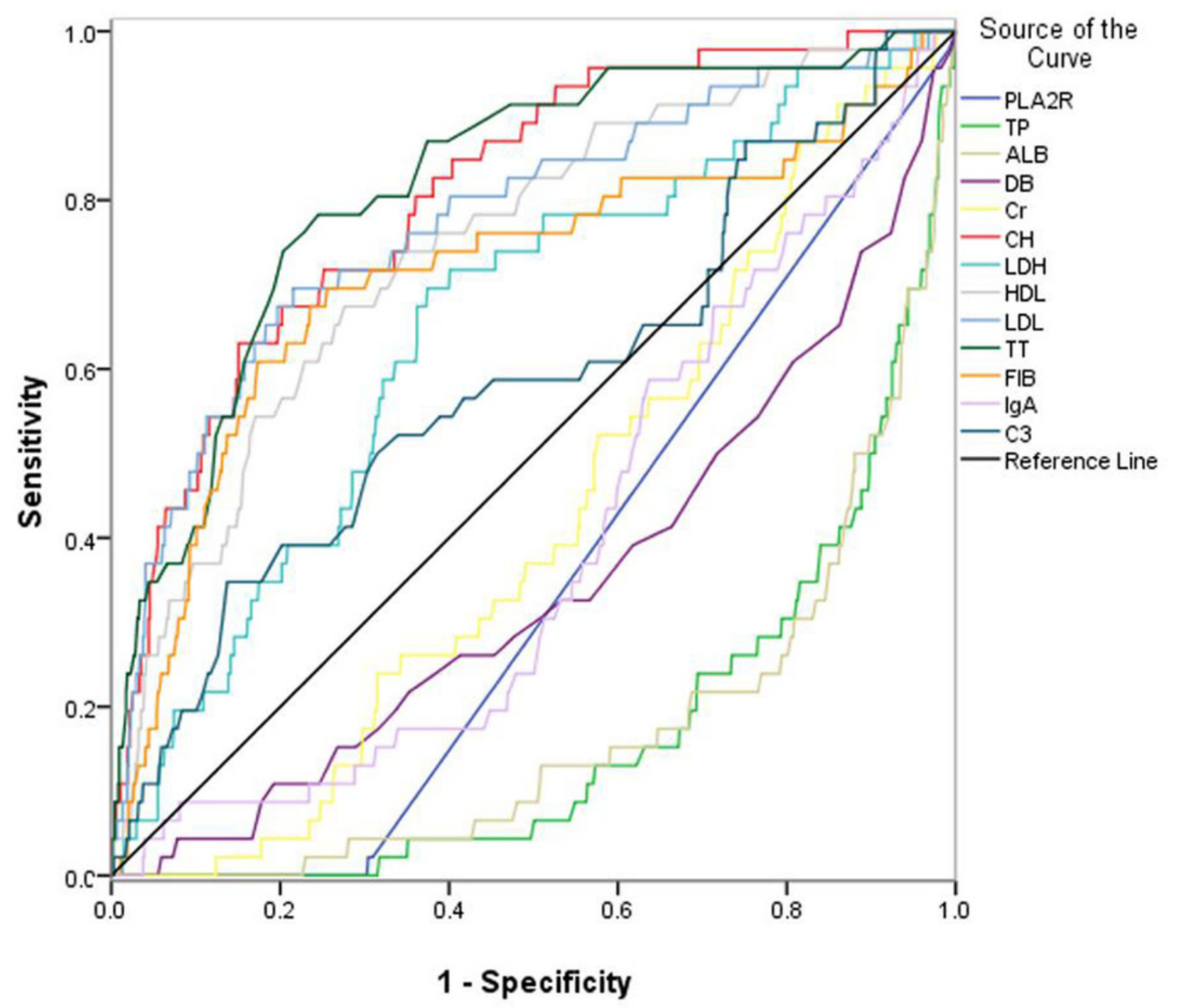


Figure 2

The ROC curve of " $\mathrm{CH}+\mathrm{T}$ " combination from logistic regression of the predicted probability for MCD patients.

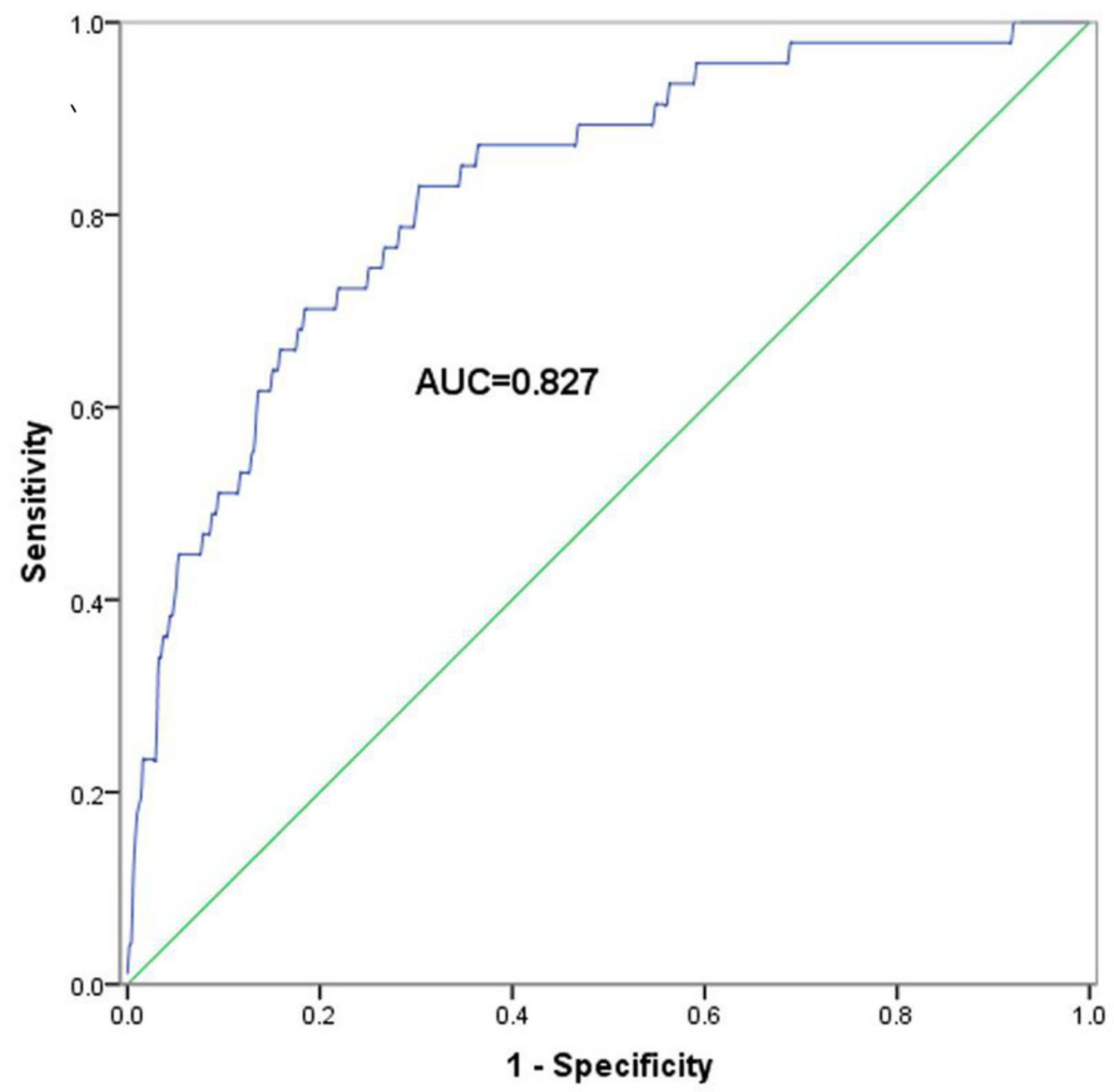

